\title{
РЕЗУЛЬТАТЫ ЛЕЧЕНИЯ РАКА ПАРАЩИТОВИДНОЙ ЖЕЛЕЗЫ
}

\author{
Северская Н.В., Ильин А.А., Исаев П.А, Полькин В.В., Желонкина Н.В., Чеботарева И.В., Дербугов Д.Н., \\ Севрюков Ф.Е.
}

МРНЦ им. А.Ф.Цыба- филиал ФГБУ «НМИЦ радиологии» Минздрава России, Обнинск

ЦЕЛЬ: представить результаты клинического обследования и лечения больных раком паращитовидной железы (ПЩЖ).

ПАЦИЕНТЫ И МЕТОДЫ: РетроспективНЫЙ анализ 16 больных с гистологически подтвержденным раком ПЩЖ, проходивших обследование и лечение с 2001 по 2019 гг. 6 мужчин, 10 женщин. Возраст на момент удаления опухоли ПЩЖ 52 (24-73) года. Медиана наблюдения после операции - 16 мес (3 мес 11,8 лет).

РЕЗУЛЬТАТЫ: размер опухоли составил 32 мм (13-45 мм). У 10 поражение правой ПЩЖ, у 6 - левой ПЩЖ. Инвазия в сосуды - 10, жировую клетчатку - 6, в щитовидную железу - 3, мышцы - 1 . Метастазы в регионарные лимфоузлы - у 1. Медиана концентрации дооперационного паратгормона (ПТГ) - 732 (136-2400) пг/мл, кальция 3,03 (2,72-3,88) ммоль/л, фосфора 0,68 (0,61-0,88) ммоль/л. Тяжелая костная форма гиперпаратиреоза с фиброзно-кистозным остеитом - у 6. Медиана ПТГ у больных с костной формой значимо выше (1543 vs 462 пг/мл, p=0,006). Объем хирургического лечения - паратиреоидэктомия (птэ) - у 10, птэ+гемитиреоидэктомия - у 2, птэ+тиреоидэктомия+ лимфодиссекция - у 4. Нормализация ПТГ и кальция после операции - у 14 (87,5\%) больных. У двоих уровень ПТГ и кальция сохранялся высоким. При ПЭТ/КТ с 18F-фторхолином у последних выявлены отдаленные метастазы в легкие и печень $(\mathrm{n}=1)$ и позвонок Th6 $(\mathrm{n}=1)$. Среди больных с нормализацией ПТГ после операции у одного возник местный рецидив через 4 года. Пациент с местным рецидивом повторно оперирован. При гистологии рак ПЩЖ с инвазией в щитовидную железу, жировую клетчатку, мышцы. Наблюдался 3 года без рецидива. Пациент с метастазом в позвонок Th6 также успешно оперирован (ламинэктомия Th6), с нормализацией ПтГ и кальция, и 1,5 года наблюдается без рецидива. Больная с метастазами в легкие и печень получала после операции сорафениб, умерла от прогрессирования заболевания через 12 мес. после постановки диагноза. У остальных пациентов после операции данных за рецидив/прогрессирование не получено.

Выводы: отдаленные метастазы выявлены у 13\% (2/16) больных раком ПщЖ и были диагностированы сразу после хирургического лечения (птэ) при сохраняющемся высоком уровне ПТГ и кальция. ПЭТ/КТ с 18F-фторхолином является информативным методом топической диагностики отдаленных метастазов рака ПЩЖ. Нормализация ПТГ после операции свидетельствует в пользу радикальности хирургического лечения рака ПЩЖ, однако не исключает вероятность местного рецидива в дальнейшем у 7\% (1/14) больных, что требует длительного динамического наблюдения. 